\title{
Targeted treatments of bone metastases in patients with lung cancer
}

\author{
Vera Hirsh* \\ McGill University Health Centre, Royal Victoria Hospital, Montreal, QC, Canada
}

Edited by:

Miguel Angel Villalona, The Ohio State

University, USA

\section{Reviewed by:}

Markus Joerger, Kantonsspital St. Gallen, Switzerland

Meng Xu Welliver, The Ohio State

University James Cancer Center, USA

\section{*Correspondence:}

Vera Hirsh, McGill University Health Centre, Royal Victoria Hospital, 687

Pine Avenue West, Montreal, OC H3A

1A1, Canada

e-mail:vera.hirsh@muhc.mcgill.ca
Until now 30-40\% of patients with advanced lung cancer develop bone metastases, but as the newer therapies are extending survival, the chance of developing bone metastases increases. Bone metastases cause skeletal-related events (SREs) such as pathologic fractures, spinal cord compression, radiation therapy or surgery to bone, or hypercalcemia, which can have debilitating consequences affecting patients' health-related quality of life (HR-OOL) and performance status (PS). Poor PS then prevents the patients to receive further lines of treatments, which are available today. SREs are associated with increased economic costs. In one clinical trial, the median time to first SRE was only 5 months. Early detection of bone metastases can prevent SREs and avoid inappropriate implementation of major surgery or chemoradiation therapy. With the new generation bisphosphonate zoledronic acid (ZA) or denosumab (anti-RANKL activity), one can reduce the number of patients who experience SREs, decrease the annual incidence of SREs and delay the median time to first SRE. These agents are effective even after the onset of SREs. They are well tolerated, with manageable side effects. The biochemical markers of bone metabolism especially Ntelopeptide of type I collagen and bone specific alkaline phosphatase (BALP) can be both prognostic and predictive markers for the patients with bone metastases from non-small cell lung cancer (NSCLC). Anticancer activity of ZA and denosumab further supports their use as soon as bone metastases are diagnosed in patients with NSCLC. Further trials will inform us about the efficacy of these agents for prevention of bone metastases and even about possible effects on visceral metastases.

Keywords: bone metastases, denosumab, zoledronic acid, NSCLC, biomarkers, skeletal-related events, effect on pain and survival

\section{INTRODUCTION}

Approximately $30-40 \%$ of patients with lung cancer develop bone metastases (1), which can lead to skeletal-related event (SREs) such as pathologic fractures, spinal cord compression, radiation therapy or surgery to bone, or hypercalcemia. These SREs can affect the patient's health-related quality of life (HR-QOL). Bone metastases are the most common cause of cancer-associated pain in patients with advanced malignancies (2). The bone pain associated with bone metastases often requires palliative radiation therapy. Pathologic fracture, which may require surgery, spinal cord compression, and hypercalcemia of malignancy (HCM) can be life-threatening. In a large prospective trial, pathologic fractures were significantly and negatively correlated with survival among 460 patients with bone metastases from solid tumors, including breast, prostate, kidney, and lung cancers (3). SREs not only cause increased morbidity and deterioration of performance status (PS), but also increased economic costs (4), thus SRE prevention will not only decrease patient morbidity, improve HR-QOL, but will also be associated with decreased use of health care resources. The need to focus on bone metastases and their sequelae is heightened as the survival of patients with non-small cell lung cancer (NSCLC) increases with the newer therapies. In one clinical trial, median time to first SRE in patients with NSCLC was 5 months only (5). To prevent SREs, preserve patients' QOL, good PS, and functional independence are of great importance and will allow patients to receive all the lines of therapies now available.

\section{PATHOPHYSIOLOGY OF BONE METASTASES}

The release of growth factors from the bone matrix during osteoclast-mediated osteolysis is conducive to the development of metastatic lesions (6). In osteolytic lesions, factors secreted by tumor cells induce osteoclast recruitment and activation, leading to increased osteolysis (7). Elevated osteolysis decreases bone integrity, can cause bone pain and the release of minerals from the bone matrix, resulting in HCM (8). Bone resorption releases growth factors that stimulate tumor growth and increase of osteoclast-stimulating factors (9). In contrast, tumor cells in osteoblastic lesions secrete factors that stimulate osteoblasts, which are responsible for the formation of new bone tissue (osteogenesis). Levels of osteolysis are enhanced in response to increased osteogenesis, releasing growth factors from the bone matrix (7). Osteoblastic lesions may also contain a strong osteolytic component that can decrease bone integrity $(9,10)$. Aberrant new bone formation in osteoblastic lesions produces new bone tissue that is abnormal, malformed, and does not add to the overall bone strength $(9,11)$. 


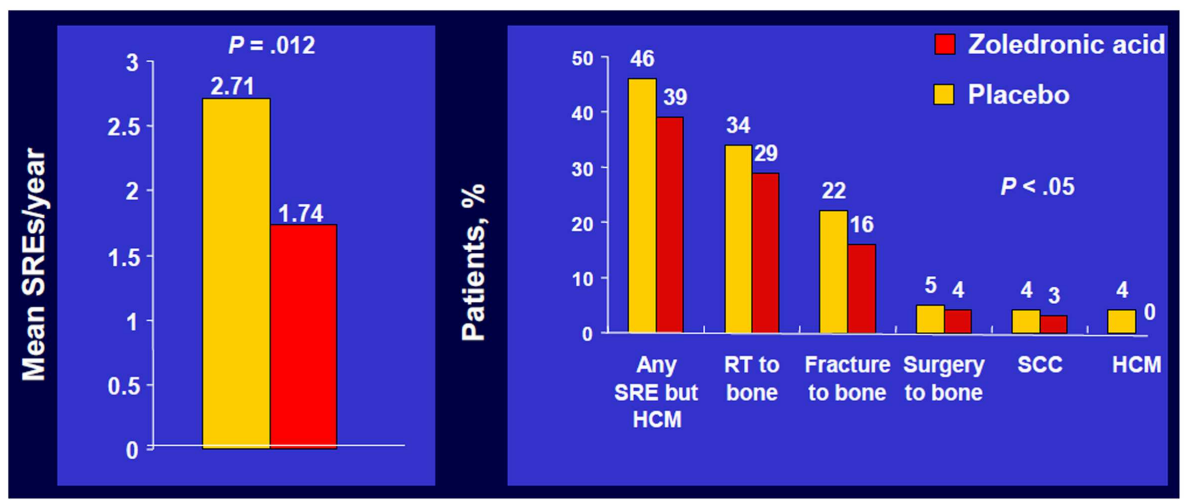

FIGURE 1 | Zoledronic Acid reduced percentage of patients with each SRE. Phase III trial of patients with bone metastases from NSCLC/OST who received ZOL or placebo every 3 weeks for up to 21 months. Approximately $50 \%$ of patients had NSCLC; $7 \%$ of patients had SCLC.
SRE, skeletal-related event; mets, metastases; NSCLC, non-small cell lung cancer; OST, other solid tumors; RT, radiotherapy; SCC, spinal cord compression; HCM, hypercalcemia of malignancy. Data from Rosen et al. (5).

\section{EARLY DETECTION OF BONE METASTASES}

The incorrect staging of patients with NSCLC can result in suboptimal treatment decisions such as major surgery or an aggressive chemoradiation without hope for a curative outcome.

Recently, PET scanning for accurate staging of NSCLC has been recognized as a valuable tool by the National Comprehensive Cancer Network (12). Fluorine-18 deoxyglucose (FDG)-PET scans for the detection of bone metastases in NSCLC have been shown to have a higher specificity compared with bone scans ( 90 versus $70 \%$, respectively) $(13,14)$ and a much lower rate of false negatives ( 6 versus 39\%, respectively) (15). The sensitivity of FDG-PET and bone scans for the detection of bone metastases from NSCLC was comparable after appropriate follow-up imaging $(13,14)$.

\section{CLINICAL IMPLICATIONS OF BONE \\ METASTASES - BISPHOSPHONATES, ZOLEDRONIC ACID}

Bisphosphonates are pyrophosphate analogs that are deposited at sites of bone remodeling. They bind to bone mineral surfaces and are ingested by osteoclasts wherein they inhibit osteolysis (16). Early bisphosphonates i.e., etidronate, clodronate, demonstrated efficacy for the treatment of HCM, but these agents are weak with limited utility in the oncology setting (16).

The introduction of a nitrogen group to the bisphosphonate backbone resulted (17) in increased potency and a different cellular target: farnesyl diphosphonate synthase, a key enzyme in the mevalonate pathway. These bisphosphonates inhibit protein prenylation and RAS signaling in osteoclasts, thereby inducing apoptosis (18). Zoledronic acid consistently achieved the greatest antiresorptive efficacy among the bisphosphonates tested in preclinical assays in human cancer cell lines and animal models $(19,20)$.

Regulatory approval for zoledronic acid (ZA) in patients with any solid tumors was based on results from a phase III randomized, placebo-controlled trial in which 773 patients with bone metastases from solid tumors other than breast or prostate cancer received ZA (4 or $8 \mathrm{mg}$ ) or placebo via $15 \mathrm{~min}$ intravenous infusion every 3 weeks for up to 21 months (5). Among the 507 patients randomized to the $4 \mathrm{mg}$ ZA or placebo group of this trial, 249 had NSCLC and 36 had small cell lung cancer (SCLC).

In the overall trial population, ZA significantly reduced the number of patients who experienced at least one SRE, including $\mathrm{HCM}, 39$ versus $48 \%$ with placebo, $p=0.039$, and reduced the proportion of patients who experienced each type of SRE (Figure 1) (5).

Zoledronic acid also significantly decreased the annual incidence of SREs, 1.74 versus 1.71 per year for placebo, $p=0.012$ and significantly delayed the median time to first SRE compared with placebo (236 versus 155 days, respectively, $p=0.009$ ) (5). A multiple event analysis using a robust Andersen-Gill model was performed for the overall population. This analysis takes into account not only the number of SREs but also the timing between the SREs, thereby providing a sensitive comparison of the ongoing risk of SREs between two treatment groups.

Zoledronic acid reduced the risk of SREs by $31 \%$ versus placebo in the overall trial population (relative risk, $\mathrm{RR}=0.693$, $p=0.003$ ). Many patients with lung cancer are diagnosed only after the first SRE. However, pre-existing skeletal morbidity does not preclude the benefits of subsequent therapy with ZA. Indeed, patients who have already experienced an SRE are at especially high risk for subsequent events. In an exploratory analysis of the ZA phase III trial in patients with NSCLC and other solid tumors, patients with a history of SRE before study entry had a $41 \%$ increased risk of experiencing an on-study SRE compared with patients with no history of prior SRE $(p=0.036)$ (21). In patients with a prior SRE, ZA produced a significant $31 \%$ reduction in the risk of developing an on-study SRE compared with placebo in a robust Andersen-Gill multiple event analysis, $p=0.009$, and significantly reduced the skeletal morbidity rate, 1.96 versus 2.81 events per year for placebo, $p=0.030(21)$.

Furthermore, ZA significantly prolonged the median time to first SRE on study by $\sim 4$ months compared with placebo in this prior-SRE cohort (215 versus 106 days, respectively, $p=0.011$ ). Benefits were also seen in the subset of patients who had not experienced a prior SRE, but without a statistical significance 
because of lack of the statistical power. This study suggests that ZA is effective and provides benefits even after the onset of SREs.

The most commonly reported adverse events (AEs) for ZA and placebo during the trial were bone pain including infusion of ZA-related pain (48 and 58\%, respectively), nausea (47 and $32 \%$, respectively), and dyspnea (45 and 30\%, respectively) (22). There was no significantly lower incidence of palliative radiotherapy to bone in the $4 \mathrm{mg}$ ZA group versus placebo (23). There were no grade 4 increases in serum creatinine in the NSCLC stratum. Monitoring of renal function and oral health during bisphosphonate therapy is recommended to avoid uncommon, but potentially serious AEs $(24,25)$. Because all intravenous bisphosphonates are cleared by the kidneys, renal function, and hydration status should be determined before each infusion to ensure renal safety. Reduced starting dose of ZA is recommended for patients with impaired renal function (26).

Osteonecrosis of the jaw (ONJ) has been reported as an uncommon event in patients receiving bisphosphonates and is characterized by exposed bone in the maxillofacial area with no evidence of healing after 6 weeks of appropriate dental care in the absence of metastatic disease or radiation to the jaw (25). The reports using the data obtained from retrospective analyses and reviews of medical records databases suggest that the frequency of ONJ in patients with malignant bone disease may be between 0.7 and $12.6 \%(27-29)$.

This wide range in ONJ frequency is likely due to variability in preventive dental measures before and during bisphosphonate therapy, variations in the duration of bisphosphonate treatment, and geographic differences. Preventive dental measures and appropriate oral hygiene have been identified that can significantly reduce the incidence of ONJ during bisphosphonate therapy (25, 30-32). A pilot study in patients with active ONJ lesions found that local application of a medical ozone oil suspension led to complete ONJ resolution (33).

\section{ZOLEDRONIC ACID AND BIOCHEMICAL MARKERS}

In a subset of patients with NSCLC or other solid tumors in the placebo group (238 patients), urinary levels of the bone resorption marker N-telopeptide of type I collagen (NTX) and the serum bone formation marker bone specific alkaline phosphatase (BALP) were assessed approximately every 3 months (34). High NTX levels ( $\geq 100 \mathrm{nmol} / \mathrm{mmol}$ creatinine) at baseline were associated with an increased risk of first SRE $(\mathrm{RR}=1.85, p=0.076)$ and bone disease progression $(\mathrm{RR}=1.76, p=0.029)$ compared with patients with low NTX levels $(<100 \mathrm{nmol} / \mathrm{mmol}$ creatinine, Figure 2) (34). Moreover, compared with patients with low NTX levels, patients with high NTX levels had a more than threefold increased risk of death $(\mathrm{RR}=3.03, p<0.001)$ and a 5 -month reduction in median survival (3.2 versus 8.2 months for patients with low baseline NTX levels) (34). Patients with high baseline BALP levels ( $\geq 146 \mathrm{IU} / \mathrm{L})$ also had statistically significant increases in risk of disease progression $(\mathrm{RR}=1.77, p=0.005)$ and death $(\mathrm{RR}=1.53, p=0.003)$ compared with patients with low BALP levels (<146 IU/L) (34).

Exploratory analysis of the ZA phase III clinical trial database (36) showed that ZA reduced mean urinary NTX levels within 3 months in patients with bone metastases from NSCLC and

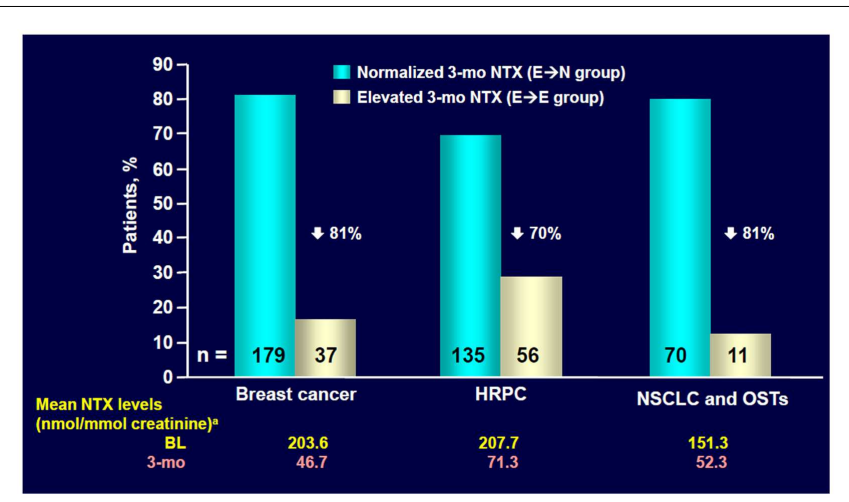

FIGURE 2 | ZOL normalized NTX levels within 3 months in most patients with elevated baseline NTX. NTX, N-telopeptide of type I collagen; HRPC, hormone-refractory prostate cancer; NSCLC, non-small cell lung cancer; OST, other solid tumors; BL, baseline. Data from Lipton et al. (35).

other solid tumors who had bone marker assessment $(n=204)$ (35). ZA also significantly reduced the RR of death by $35 \%$ versus placebo $(\mathrm{RR}=0.650, p=0.024)$ among patients with NSCLC and high baseline NTX levels (NTX $\geq 64 \mathrm{nmol} / \mathrm{mmol}$ creatinine, $n=144$ ) (37).

Differences in survival between the ZA and placebo groups did not reach statistical significance in the normal baseline NTX subset, consistent with the lower risks of SREs and death that have been reported for that subset $(34,37)$.

This benefit could result from reduced osteolysis, resulting in less release of growth factors from the bone matrix, reduced SRE rate or possibly also from direct and indirect antitumor effects of ZA i.e., increased apoptosis, synergism with chemotherapy, antiangiogenesis, and stimulation of immune system.

\section{ANTICANCER ACTIVITY OF ZOLEDRONIC ACID}

There is a preclinical evidence that ZA can inhibit proliferation and induce apoptosis in a broad range of human cancer cell lines (16, 38) ZA also exerts antitumor synergy with chemotherapy agents in the A549 lung cancer cell line $(39,40)$. In murine lung cancer cell line, ZA inhibited the growth of these tumors and mice treated with ZA survived significantly longer than the untreated mice $(p<0.05)(41)$.

Multiple effects may contribute to the antitumor activity of ZA that has been reported in preclinical models (42). In addition to direct antitumor effects, nitrogen-containing bisphosphonates appear to have immunomodulatory properties especially with regard to $\gamma \delta \mathrm{T}$ cells, a subset of $\mathrm{T}$ cells that plays a role in immunosurveillance for malignancies. In an in vitro model, ZA induced maturation and upregulated co-stimulating surface receptor expression (e.g., CD 40, CD 80, CD 83) on peripheral $\gamma \delta$ $\mathrm{T}$ cells (43). In addition, bisphosphonates have been shown to activate the cytolytic activity of $\gamma \delta$ T cells and therefore, may enhance the antitumor immune response (44).

There are ongoing clinical studies in patients with NSCLC evaluating the efficacy of ZA both for prevention of bone metastases and for antitumor activity. 


\section{DENOSUMAB AND ANTI-RANKL ACTIVITY}

Denosumab is a fully human monoclonal antibody that binds to and neutralizes RANKL (receptor activator of nuclear factor kappa-B ligand) thereby inhibiting osteoclast function and preventing generalized bone resorption and local bone destruction.

It is hypothesized that tumor cells in the bone lead to increased expression of RANKL on osteoclasts and their precursors. RANKL is an essential mediator of osteoclast function, formation, and survival (45-47). Excessive RANKL-induced osteoclast activity results in resorption and local bone destruction with evidence of elevated levels of bone turnover markers, leading to SREs $(34,36)$.

Denosumab has been studied in two phase II trials of patients with bone metastases in advanced cancer and in one phase II trial with myeloma (48-50). These studies demonstrated that treatment with denosumab at doses ranging from 30 to $180 \mathrm{mg}$ administered every 4 or 12 weeks was associated with a rapid and sustained suppression of bone turnover markers and delay of SREs similar to that seen with i.v. bisphosphonates.

In a randomized, double-blind phase III trial of denosumab versus $\mathrm{ZA}$, in the treatment of bone metastases in patients with advanced cancer (excluding breast and prostate cancer) or multiple myeloma, 1779 patients were enrolled onto study, 890 patients analyzed on ZA, 886 on denosumab (51). Baseline characteristics were well balanced (Table 1). The primary endpoint was time to first on-study SRE comparing denosumab with ZA for noninferiority. Secondary efficacy endpoints were to be evaluated only if non-inferiority was demonstrated, and were superiority tests comparing denosumab and ZA for time to first on-study SRE and time to first and subsequent SRE by multiple event analysis. A subsequent SRE was defined as an event occurring $\geq 21$ days after the previous SRE.

The median number of doses was seven for ZA and seven for denosumab with cumulative drug exposure of 651.9 patient-years for ZA and 675.3 patient-years for denosumab. Median time on study was $\sim 7$ months.

Table 1 | Baseline characteristics.

\begin{tabular}{lcc}
\hline $\begin{array}{l}\text { Characteristic, } \boldsymbol{n}(\%) \\
\text { or median }\end{array}$ & $\begin{array}{c}\text { Zoledronic acid } \\
(\boldsymbol{n}=\mathbf{8 9 0 )}\end{array}$ & $\begin{array}{c}\text { Denosumab } \\
(\boldsymbol{n}=\mathbf{8 8 6})\end{array}$ \\
\hline Male & $552(62)$ & $588(66)$ \\
Age (years) & 61 & 60 \\
Primary tumor type & $345(39)$ & $343(39)$ \\
$\quad$ Non-small cell lung cancer & $93(10)$ & $86(10)$ \\
$\quad$ Multiple myeloma & $452(51)$ & $457(52)$ \\
Other & $728(82)$ & $748(84)$ \\
ECOG performance status of 0 or 1 & 2 & 2 \\
Time from first bone metastasis to & & $440(50)$ \\
randomization (months) & $446(50)$ & $474(53)$ \\
Previous SRE & $448(50)$ & \\
Presence of visceral metastases & & \\
\hline
\end{tabular}

See Ref. (52).
Denosumab was non-inferior to ZA in delaying time to first onstudy SRE (HR $=0.84, p=0.0007)$ representing $16 \%$ reduction in hazard (Figure 3). The median time to first on-study SRE was 20.6 months for denosumab and 16.3 months for ZA. The test for superiority for time to first SRE showed $p=0.06$ and therefore did not reach statistical significance. Time to first and subsequent SREs (multiple events) analysis demonstrated a rate ratio of 0.90 for denosumab compared with ZA, $p=0.14$, which was not statistically significant. Overall survival $(\mathrm{HR}=0.95, p=0.43)$ and disease progression ( $\mathrm{HR}=1.00, p=1.0)$ were similar between treatment groups (Figures $\mathbf{4}$ and $\mathbf{5}$ ).

The effect of denosumab on time to first on-study SRE relative to ZA by tumor stratification factors resulted in an $\mathrm{HR}=0.84$ for NSCLC, $p=0.20 ; 1.03$ for myeloma, $p=0.89$, and 0.79 for other solid tumors, $p=0.04$. An ad hoc analysis examining overall survival demonstrated an $\mathrm{HR}=0.79$ for NSCLC, 2.26 for myeloma, and 1.08 for other solid tumors.

Patients in both arms experienced similar rates of AEs (Table 2). Rates of serious AEs are $13.4 \%$ for ZA versus $14.6 \%$ for denosumab. New primary malignancy occurred in three patients $(0.3 \%)$ receiving $\mathrm{ZA}$ and in five patients $(0.6 \%)$ receiving denosumab.

Adverse events of hypocalcemia occurred more frequently with denosumab ( $10.8 \%$ denosumab, $5.8 \% \mathrm{ZA})$. In general, the clinical consequences of hypocalcemia were not observed. Centrally determined grade 3 and 4 decreases in albumin-adjusted calcium values were reported in 9 patients (1\%) receiving ZA and 20 patients $(2.3 \%)$ receiving denosumab. IV calcium was administered on study to $2.7 \%$ of patients receiving ZA and $5.7 \%$ receiving denosumab.

Positive adjudicated ONJ occurred with cumulative incidence rates in the $\mathrm{ZA}$ and denosumab groups of 0.6 and $0.5 \%$ at 1 year, respectively, 0.9 and $1.1 \%$ at 2 years, and 1.3 and $1.1 \%$ at 3 years $(p=1.0)$.

Adverse events associated with acute phase reactions within the first 3 days after dose 1 occurred in $14.5 \%$ of patients receiving $\mathrm{ZA}$ versus $6.9 \%$ receiving denosumab. Most frequent reactions were pyrexia, arthralgia, and fatigue. One hundred fifty-two patients $(17.3 \%)$ on ZA required dose adjustments to levels lower than $4 \mathrm{mg}$ and doses were withheld because of elevated serum creatinine in 78 patients $(8.9 \%)$. No dose adjustments or dose withholding for renal function were required for denosumab. Despite appropriate adjustments of the ZA dosing regimen for renal function, there was an evidence of an excess of renal AEs with ZA. Denosumab has no limitations with respect to renal impairment as it is a monoclonal antibody and is eliminated by intracellular catabolism in phagocytes, with no evidence of renal effects $(53,54)$.

\section{BONE TURNOVER BIOMARKERS - DENOSUMAB VERSUS ZOLEDRONIC ACID}

Patients treated with denosumab experienced a greater suppression of bone turnover markers than with ZA. Between baseline and study week 13 levels of urinary NTX/Cr decreased by a median of $76 \%$ for denosumab $(n=546)$ and $65 \%$ for ZA $(n=543)$, $p<0.001$ and BALP decreased by $37 \%$ for denosumab $(n=578)$ and $29 \%$ for ZA $(n=581), p<0.001$. 


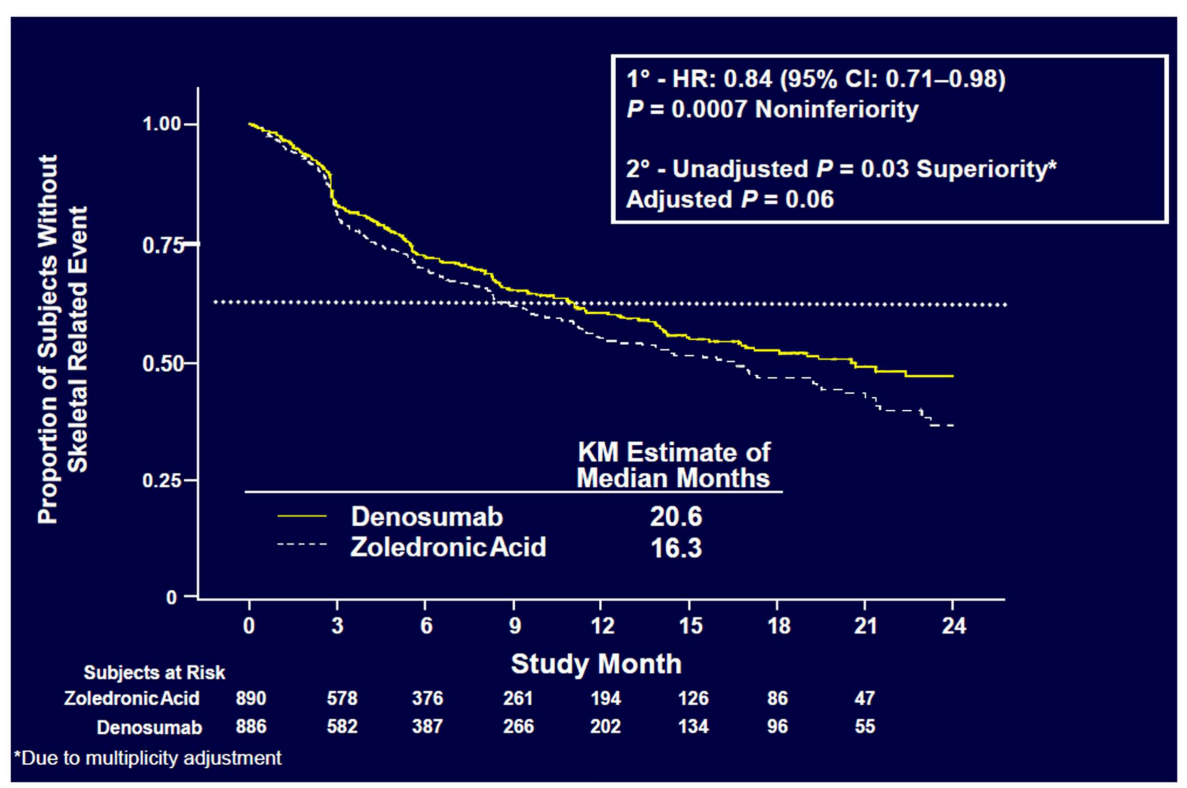

FIGURE 3 |Time to first on-study SRE (52).

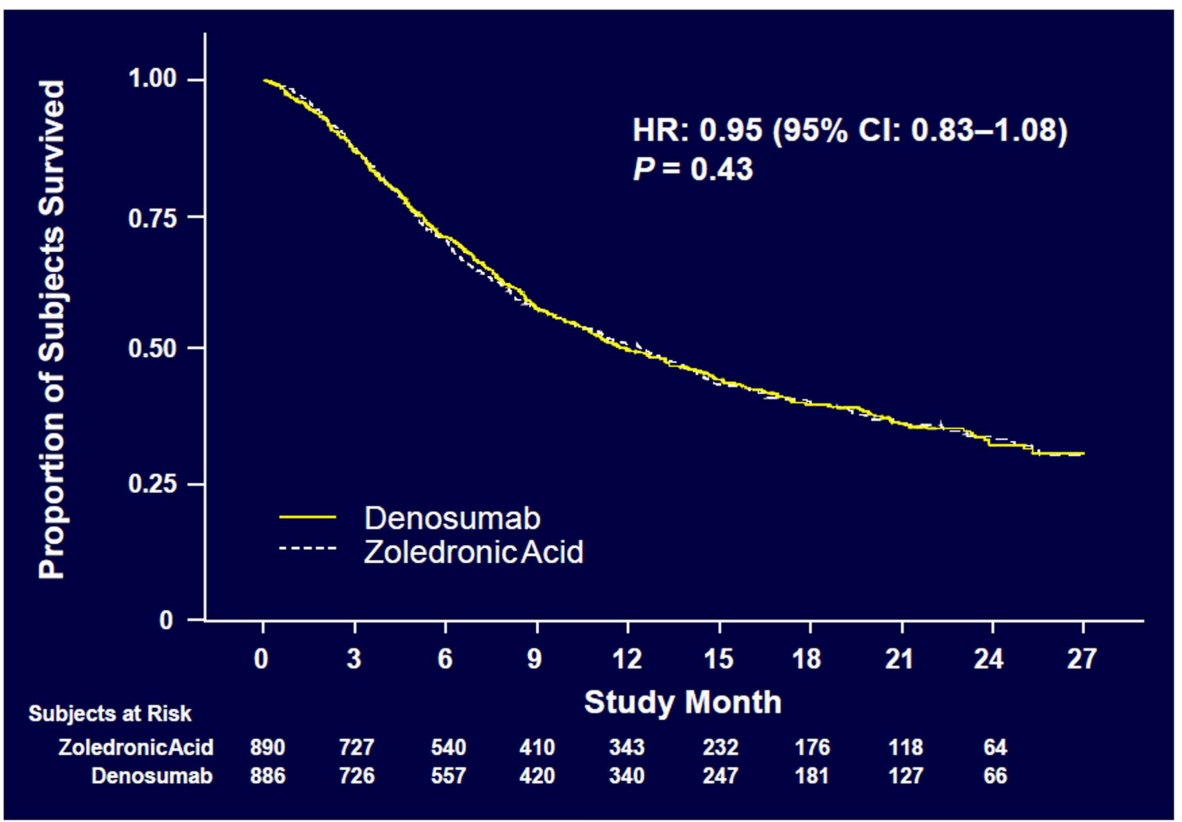

FIGURE 4 | Overall survival (52).

\section{EXPLORATORY ANALYSIS OF OVERALL SURVIVAL IN LUNG CANCER}

Sub-analysis of 811 patients with any lung cancer showed that denosumab was associated with significantly improved overall median survival compared with $\mathrm{ZA}$, with a difference of 1.2 months (KM median $=8.9$ versus 7.7 months, $\mathrm{HR}=0.80$, $p=0.01$ ) (Figure 6) (55). Denosumab continued to show a significant survival advantage over ZA when overall survival was adjusted for relevant baseline covariates (age, sex, time from diagnosis of primary cancer to first evidence of metastasis or the first bone metastasis, visceral metastasis, and ECOG status) and stratified by the randomization stratification factors (previous SRE and systemic anticancer therapy), $\mathrm{HR}=0.81, p=0.01$. In patients with visceral metastases (231 in denosumab group and 233 in 


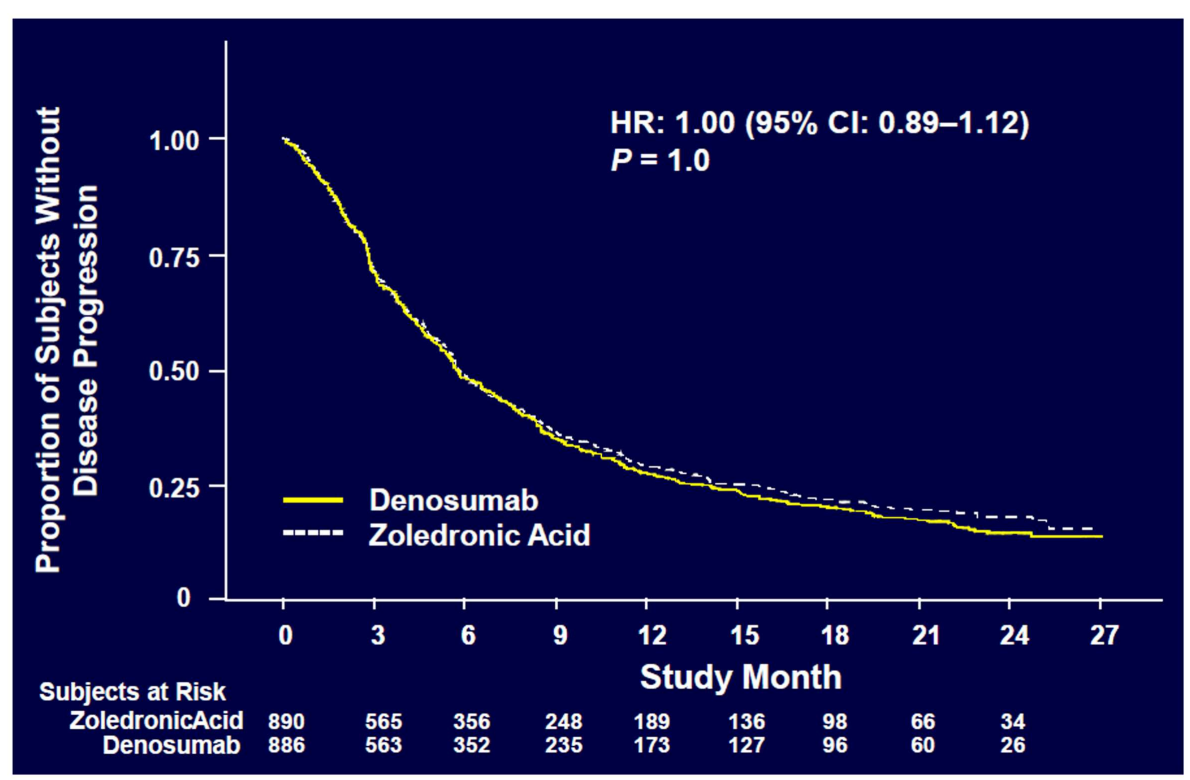

FIGURE 5 | Overall disease progression (52).

Table 2 |Adverse events of interest.

\begin{tabular}{lcc}
\hline Event, $\boldsymbol{n}(\%)$ & $\begin{array}{c}\text { Zoledronic acid } \\
(\boldsymbol{n}=\mathbf{8 7 8})\end{array}$ & $\begin{array}{c}\text { Denosumab } \\
\text { ( } \boldsymbol{n}=\mathbf{8 7 8 )}\end{array}$ \\
\hline Infectious AEs & $349(39.7)$ & $358(40.8)$ \\
Infectious serious AEs & $118(13.4)$ & $128(14.6)$ \\
Acute phase reaction (first 3 days) & $127(14.5)$ & $61(6.9)$ \\
Potential renal toxicity AEs ${ }^{a}$ & $96(10.9)$ & $73(8.3)$ \\
$\quad$ Renal failure & $25(2.8)$ & $20(2.3)$ \\
Acute renal failure & $16(1.8)$ & $11(1.3)$ \\
Cumulative rates of ONJ* & $11(1.3)$ & $10(1.1)$ \\
Year 1 & $5(0.6)$ & $4(0.5)$ \\
Year 2 & $8(0.9)$ & $10(1.1)$ \\
New primary malignancy & $3(0.3)$ & $5(0.6)$
\end{tabular}

ancludes blood creatinine increased, renal failure, renal failure acute, proteinuria, blood urea increased, renal impairment, urine output decreased, anuria, oliguria, azotemia, hypercreatininemia, creatinine renal clearance decreased, renal failure chronic, blood creatinine abnormal.

${ }^{*} p=1.0$.

No neutralizing anti-denosumab antibodies were detected.

See Ref. (52).

ZA group), denosumab was also associated with improved overall median survival with a difference of 1.2 months $(\mathrm{KM}$ median $=7.7$ versus 6.4 months, $\mathrm{HR}=0.79, p=0.03$ ). Denosumab was associated with significantly improved survival in patients with NSCLC with a difference of 1.5 months (KM median $=9.5$ versus 8.1 months, $\mathrm{HR}=0.78, p=0.01$ ) (Figure 7).

Explanation for the longer survival with the denosumab treatment in these lung cancer patients includes both direct and indirect effects on tumor cells. An indirect effect may derive from the symbiotic relationship between tumor cells and the bone marrow microenvironment in which both bone destruction and tumor growth are promoted. Tumor cells secrete various factors that stimulate production of RANKL (45). The increased expression of RANKL in the tumor environment leads to increased formation, activation, and survival of osteoclasts and results in osteolytic lesions (56). Osteolysis then results in the release of growth factors derived from bone $(45,57)$.

These growth factors increase the production of parathyroid hormone-related protein or promote tumor growth directly (45). Bone destruction increases local extracellular calcium concentrations, which have also been shown to promote tumor growth and the production of parathyroid hormone-related protein (57). Denosumab may indirectly affect skeletal tumor progression by targeting osteoclasts and disrupting the interaction between tumor cells and the bone microenvironment. RANKL inhibition has been shown to reduce bone lesions/osteolysis, and skeletal tumor burden in a model of NSCLC (58) and to enhance antitumor efficacy of other therapies on skeletal tumors $(59,60)$.

Another hypothesis is that denosumab may improve survival by directly inhibiting RANKL on RANK-expressing tumor cells, which has been demonstrated for breast cancer cells in vivo (61) and for a number of tumor cell lines (including lung cancer cells) in vitro (62). RANKL inhibition may have a direct antineoplastic effect on lung cancer cells via apoptosis or anti-migration activity (63). The hypothesis of mechanism of anticancer activities, which inhibit RANKL or RANK-expressing tumor cells has been described in more detail in the review article of Peters and Meylan (64). These findings warrant further prospective clinical investigations, denosumab might have anticancer effects beyond the skeleton (65). 


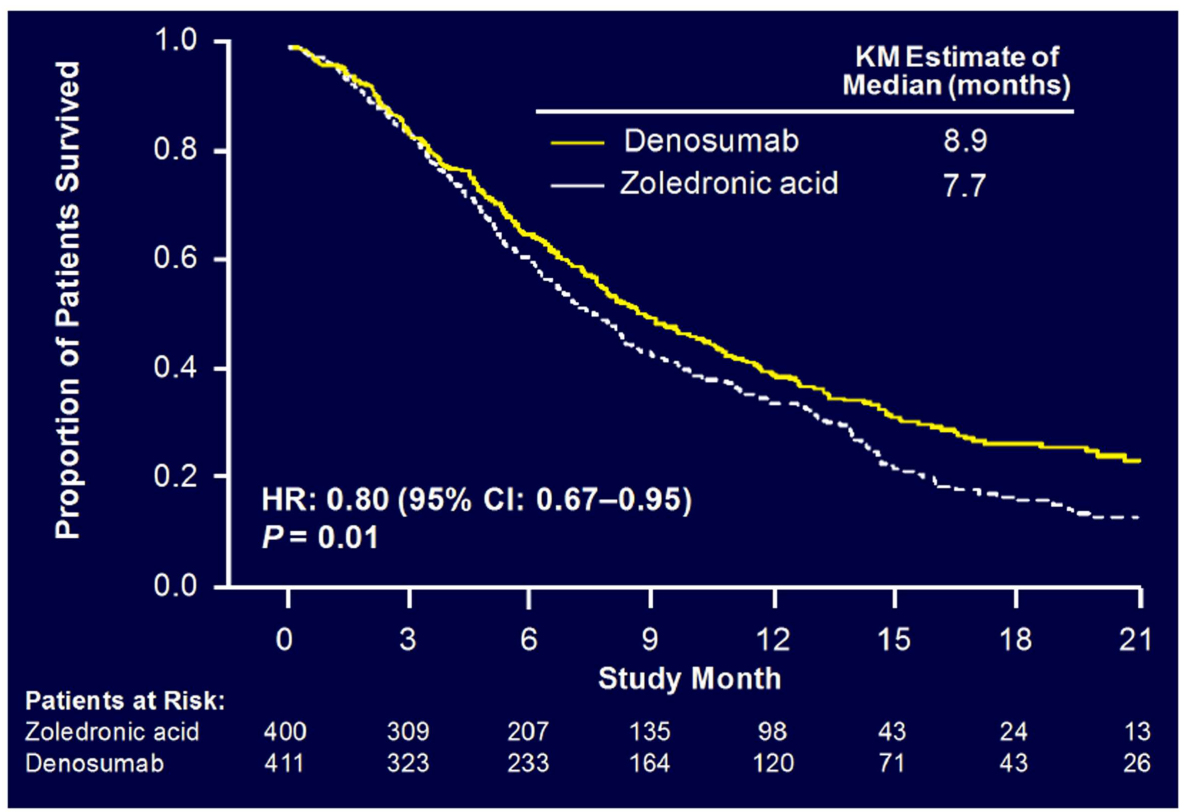

FIGURE 6 | Overall survival: patients with lung cancer.

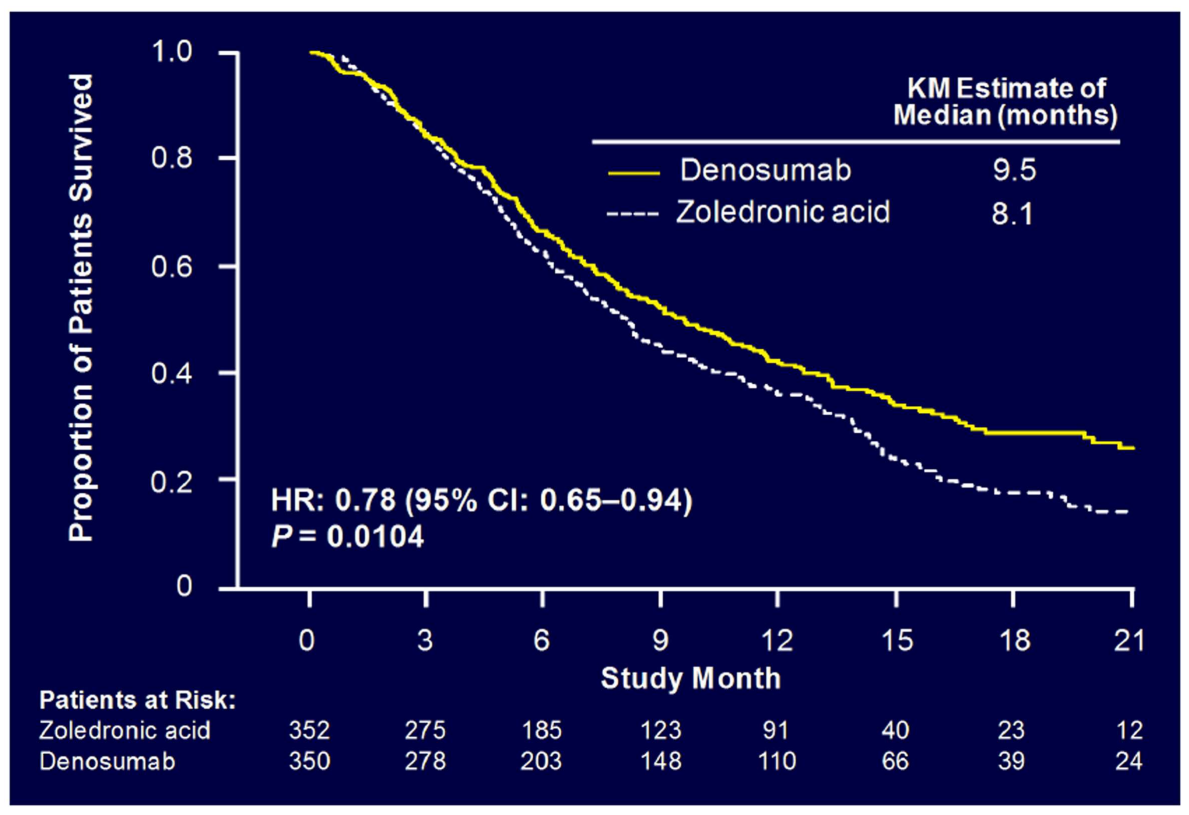

FIGURE 7 | Overall survival: patients with NSCLC

\section{PROMISING NEW BONE TARGETING AGENTS}

Targeting bone agents in the early stage of investigation in NSCLC are Dasatinib (i.e., anti-src activity) (66), ACE-011 (Sotatercept Activin TRAP) $(67,68)$, Cabozantinib (anti-RET agent) (69), and Radium 223 (targeted alpha emitter) (70).

\section{CONCLUSION}

In the palliative group of patients with metastatic lung cancer, the HR-QOL is extremely important. Preserving a good PS, which enables these patients to receive all the available lines of treatment for metastatic NSCLC is also desirable. 
Early identification of bone metastases and management of SREs have become crucial for maintaining QOL and containing healthcare costs throughout the patient's care. The identification of risk factors for skeletal metastases in patients with NSCLC will help us to implement early treatment to prevent or delay the onset of debilitating SREs.

The safety profile for ZA and denosumab is similar but subcutaneous administration of denosumab offers advantages over intravenous administration with no need for renal monitoring. Denosumab is associated with fewer acute phase reactions, but has a higher incidence of hypocalcemia. ONJ is similar for both agents. Thus both agents are a reasonable option for targeted bone therapy.

Future trials are needed to inform us about efficacy of these agents for prevention of bone metastases and effects on visceral metastases, too, thus contributing to a longer survival in patients with metastatic NSCLC.

\section{REFERENCES}

1. Coleman RE. Skeletal complications of malignancy. Cancer (1997) 80(Suppl):1588-94. doi:10.1002/(SICI)1097-0142(19971015)80:8+<1588: :AID-CNCR9>3.3.CO;2-Z

2. Mercadante S. Malignant bone pain: pathophysiology and treatment. Pain (1997) 69:1-18. doi:10.1016/S0304-3959(96)03267-8

3. Hansen BH, Keller J, Laitinen M, Berg P, Skjeldal S, Trovik C, et al. The Scandinavian Sarcoma Group Skeletal Metastasis Register. Survival after surgery for bone metastases in the pelvis and extremities. Acta Orthop Scand Suppl (2004) 75:11-5. doi:10.1080/00016470410001708270

4. Delea TE, McKiernan JM, Liss M, Edelsberg JS, Brandman J, Sung JC, et al. Impact of skeletal complications on total medical care costs in lung cancer patients with bone metastases [abstract]. J Clin Oncol (2004) 22(Suppl 14):6064.

5. Rosen LS, Gordon D, Tchekmedyian NS, Yanagihara R, Hirsh V, Krzakowski M, et al. Long-term efficacy and safety of zoledronic acid in the treatment of skeletal metastases in patients with non-small cell lung carcinoma and other solid tumors: a randomized, phase III, double-blind, placebo-controlled trial. Cancer (2004) 100:2613-21. doi:10.1002/cncr.20308

6. Mundy GR. Mechanisms of bone metastasis. Cancer (1997) 80(Suppl):1546-56. doi:10.1002/(SICI)1097-0142(19971015)80:8+<1546::AID-CNCR4>3.3.CO; 2-R

7. Saad F, Schulman CC. Role of bisphosphonates in prostate cancer. Eur Urol (2004) 45:26-34. doi:10.1016/j.eururo.2003.10.003

8. Coleman RE. Metastatic bone disease: clinical features, pathophysiology, and treatment strategies. Cancer Treat Rev (2001) 27:165-76. doi:10.1053/ctrv.2000. 0210

9. Kakonen SM, Mundy GR. Mechanisms of osteolytic bone metastases in breast carcinoma. Cancer (2003) 97(Suppl):834-9. doi:10.1002/cncr.11132

10. Coleman RE. Bisphosphonates: clinical experience. Oncologist (2004) 9(Suppl 4):14-27. doi:10.1634/theoncologist.9-90004-14

11. Lipton A. Pathophysiology of bone metastases: how this knowledge may lead to therapeutic intervention [Review]. J Support Oncol (2004) 2(3):205-13.

12. National Comprehensive Cancer Network. NCCN clinical practice guidelines in oncology: non-small cell lung cancer. (2008). Available from: http://www.nccn. org/professionals/physician_gls/PDF/nsclc.pdf

13. Bury T, Barreto A, Daenen F, Barthelemy N, Ghaye B, Rigo P. Fluorine-18 deoxyglucose positron emission tomography for the detection of bone metastases in patients with non-small cell lung cancer. Eur J Nucl Med (1998) 25:1244-7. doi:10.1007/s002590050291

14. Gayed I, Vu T, Johnson M, Macapinlac H, Podoloff D. Comparison of bone and 2-deoxy-2-[18F] fluoro-D-glucose positron emission tomography in the evaluation of bony metastases in lung cancer. Mol Imaging Biol (2003) 5:26-31. doi:10.1016/S1536-1632(03)00036-2

15. Hetzel M, Arslandemir C, König HH, Buck AK, Nüssle K, Glatting G, et al. F-18 NaF PET for detection of bone metastases in lung cancer: accuracy, costeffectiveness, and impact on patient management. J Bone Miner Res (2003) 18:2206-14. doi:10.1359/jbmr.2003.18.12.2206
16. Green JR. Preclinical profile and anticancer potential of zoledronic acid. In: Birch EV editor. Trends in Bone Cancer Research. (Vol. 24), New York: Nova Science Publishers Inc (2006). p. 217-45.

17. Fleisch H. Development of bisphosphonates. Breast Cancer Res (2002) 4:30-4. doi: $10.1186 /$ bcr487

18. Green JR. Bisphosphonates: preclinical review. Oncologist (2004) 9(Suppl 4):3-13. doi:10.1634/theoncologist.9-90004-3

19. Green J. Zoledronate: the preclinical pharmacology. Br J Clin Pract Suppl (1996) 87:16-8.

20. Green JR, Muller K, Jaeggi KA. Preclinical pharmacology of CGP 42'446, a new, potent, heterocyclic bisphosphonate compound. J Bone Miner Res (1994) 9:745-51. doi:10.1002/jbmr.5650090521

21. Hirsh V, Tchekmedyian NS, Rosen LS, Zheng M, Hei YJ. Clinical benefit of zoledronic acid in patients with lung cancer and other solid tumors: analysis based on history of skeletal complications. Clin Lung Cancer (2004) 6(3):170-4. doi:10.3816/CLC.2004.n.030

22. Bukowski R, Rosen L, Gordon D, et al. Long-term therapy with zoledronic acid is effective and safe in reducing the risk of skeletal complications in patients with bone metastases from non-small cell lung cancer (NSCLC) [poster]. Proceedings of the 10th World Conference on Lung Cancer (WCLC); 2003 Aug 10-14. Vancouver (2003).

23. Rosen LS, Gordon D, Tchekmedyian S, Yanagihara R, Hirsh V, Krzakowski $\mathrm{M}$, et al. Zoledronic acid versus placebo in the treatment of skeletal metastases in patients with lung cancer and other solid tumors: a phase III, doubleblind, randomized trial - the Zoledronic Acid Lung Cancer and other Solid Tumors Study Group. J Clin Oncol (2003) 21:3150-7. doi:10.1200/JCO. 2003.04.105

24. Conte P, Guarneri V. Safety of intravenous and oral bisphosphonates and compliance with dosing regimens. Oncologist (2004) 9(Suppl 4):28-37. doi:10.1634/ theoncologist.9-90004-28

25. Weitzman R, Sauter N, Eriksen EF, Tarassoff PG, Lacerna LV, Dias R, et al. Critical review: updated recommendations for the prevention, diagnosis, and treatment of osteonecrosis of the jaw in cancer patients - May 2006. Crit Rev Oncol Hematol (2007) 62:148-52. doi:10.1016/j.critrevonc.2006.12.005

26. Zometa (Zoledronic acid) Injection, sterile solution for IV use, $4 \mathrm{mg} / 100 \mathrm{ml}$, Monograph Citation NDA021223, Chemical Ingredient: Zoledronic Acid Anhydrous. East Hanover, NJ: Novartis Pharmaceuticals Corporation (2005).

27. Durie BG, Katz M, Crowley J. Osteonecrosis of the jaw and bisphosphonates. $N$ Engl J Med (2005) 353:99-102. doi:10.1056/NEJM200507073530120

28. Hoff AO, Toth BB, Altundag K, Johnson MM, Warneke CL, Hu M, et al. Frequency and risk factors associated with osteonecrosis of the jaw in cancer patients treated with intravenous bisphosphonates. J Bone Miner Res (2008) 23:826-36. doi:10.1359/jbmr.080205

29. Pozzi S, Marcheselli R, Sacchi S, Baldini L, Angrilli F, Pennese E, et al. Bisphosphonate-associated osteonecrosis of the jaw: a review of 35 cases and an evaluation of its frequency in multiple myeloma patients. Leuk Lymphoma (2007) 48:56-64. doi:10.1080/10428190600977690

30. Dimopoulos MA, Kastritis E, Bamia C, Melakopoulos I, Gika D, Roussou M, et al. Reduction of osteonecrosis of the jaw (ONJ) after implementation of preventive measures in patients with multiple myeloma treated with zoledronic acid. Ann Oncol (2009) 20:117-20. doi:10.1093/annonc/mdn554

31. Montefusco V, Gay F, Spina F, Miceli R, Maniezzo M, Teresa Ambrosini $\mathrm{M}$, et al. Antibiotic prophylaxis before dental procedures may reduce the incidence of osteonecrosis of the jaw in patients with multiple myeloma treated with bisphosphonates. Leuk Lymphoma (2008) 49:2156-62. doi:10.1080/ 10428190802483778

32. Ripamonti CI, Maniezzo M, Campa T, Fagnoni E, Brunelli C, Saibene G, et al. Decreased occurrence of osteonecrosis of the jaw after implementation of dental preventive measures in solid tumour patients with bone metastases treated with bisphosphonates. The experience of the National Cancer Institute of Milan. Ann Oncol (2009) 20:137-45. doi:10.1093/annonc/mdn526

33. Ripamonti C, Maniezzo M, Ghiringhelli R, et al. Medical oil suspension applications heal osteonecrosis of the jaw (ONJ) in patients treated with bisphosphonates (BPs): preliminary results of a single institution protocol [poster]. Primary therapy of early breast cancer. Proceedings of the 11th International Conference [Poster 194] (2009).

34. Brown JE, Cook RJ, Major P, Lipton A, Saad F, Smith M, et al. Bone turnover markers as predictors of skeletal complications in prostate cancer, lung cancer, 
and other solid tumors. J Natl Cancer Inst (2005) 97:59-69. doi:10.1093/jnci/ dji002

35. Lipton A, Cook R, Saad F, Major P, Garnero P, Terpos E, et al. Normalization of bone markers is associated with improved survival in patients with bone metastases from solid tumors and elevated bone resorption receiving zoledronic acid. Cancer (2008) 113:193-201. doi:10.1002/cncr.23529

36. Coleman RE, Major P, Lipton A, Brown JE, Lee KA, Smith M, et al. Predictive value of bone resorption and formation markers in cancer patients with bone metastases receiving the bisphosphonate zoledronic acid. J Clin Oncol (2005) 23:4925-35. doi:10.1200/JCO.2005.06.091

37. Hirsh V, Major PP, Lipton A, Cook RJ, Langer CJ, Smith MR, et al. Zoledronic acid and survival in patients with metastatic bone disease from lung cancer and elevated markers of osteoclast activity. J Thorac Oncol (2008) 3:228-36. doi:10.1097/JTO.0b013e3181651c0e

38. Green JR. Antitumor effects of bisphosphonates. Cancer (2003) 97(Suppl):840-7. doi:10.1002/cncr.11128

39. Ozturk OH, Bozcuk H, Burgucu D, Ekinci D, Ozdogan M, Akca S, et al. Cisplatin cytotoxicity is enhanced with zoledronic acid in A549 lung cancer cell line: preliminary results of an in vitro study. Cell Biol Int (2007) 31:1069-71. doi:10.1016/j.cellbi.2007.02.004

40. Gjyrezi A, O’Brate A, Chanel-Vos C, Giannakakou P. Zoledronic acid synergizes with Taxol in an HDAC6-dependant manner: novel mechanistic implications for combination anticancer therapy with taxanes [Poster - Anti-Tubulin Agents]. The 98th AACR Annual Meeting; Apr 14-18; Los Angeles, CA (2007). Abstract 1425.

41. Li YY, Chang JW, Chou WC, Liaw CC, Wang HM, Huang JS, et al. Zoledronic acid is unable to induce apoptosis but slows tumor growth and prolongs survival for non-small cell lung cancers. Lung Cancer (2008) 59:180-91. doi:10.1016/j.lungcan.2007.08.026

42. Matsumoto S, Kimura S, Segawa H, Kuroda J, Yuasa T, Sato K, et al. Efficacy of the third-generation bisphosphonate, zoledronic acid alone and combined with anti-cancer agents against small cell lung cancer cell lines. Lung Cancer (2005) 47:31-9. doi:10.1016/j.lungcan.2004.06.003

43. Landmeier S, Altvater B, Pscherer S, Varnholt L, Bollard CM, Juergens H, et al. Presentation of Epstein Barr virus (EBV) epitopes by activated human $\gamma \delta \mathrm{T}$ cells induces peptide-specific cytolytic CD8+ T cell expansion [Poster Session \# 866-I]. Blood (2006) 108:1738.

44. Fournier PG, Chirgwin JM, Guise TA. New insights into the role of T cells in the vicious cycle of bone metastases. Curr Opin Rheumatol (2006) 18:396-404. doi:10.1097/01.bor.0000231909.35043.da

45. Roodman GD. Mechanisms of bone metastasis. N Engl J Med (2004) 350:1655-64. doi:10.1056/NEJMra030831

46. Hofbauer LC, Neubauer A, Heufelder AE. Receptor activator of nuclear factorkappa B ligand and osteoprotegerin: potential implications for the pathogenesis and treatment of malignant bone diseases. Cancer (2001) 92:460-70. doi:10.1002/1097-0142(20010801)92:3<460::AID-CNCR1344>3.0.CO;2-D

47. Selvaggi G, Scagliotti GV. Management of bone metastases in cancer: a review. Crit Rev Oncol Hematol (2005) 56:365-78. doi:10.1016/j.critrevonc. 2005.03.011

48. Body JJ, Facon T, Coleman RE, Lipton A, Geurs F, Fan M, et al. A study of the biological receptor activator of nuclear factor-kappa B ligand inhibitor, denosumab, in patients with multiple myeloma or bone metastases from breast cancer. Clin Cancer Res (2006) 12:1221-8. doi:10.1158/1078-0432.CCR-05-1933

49. Fizazi K, Lipton A, Mariette X, Body JJ, Rahim Y, Gralow JR, et al. Randomized phase II trial of denosumab in patients with bone metastases from prostate cancer, breast cancer, or other neoplasms after intravenous bisphosphonates. J Clin Oncol (2009) 27:1564-71. doi:10.1200/JCO.2008.19.2146

50. Lipton A, Steger GG, Figueroa J, Alvarado C, Solal-Celigny P, Body JJ, et al. Randomized active-controlled phase II study of denosumab efficacy and safety in patients with breast cancer-related bone metastases. J Clin Oncol (2007) 25:4431-7. doi:10.1200/JCO.2007.11.8604

51. Henry DH, Costa L, Goldwasser F, Hirsh V, Hungria V, Prausova J, et al. Randomized, double-blind study of denosumab versus zoledronic acid in the treatment of bone metastases in patients with advanced cancer (excluding breast and prostate cancer) or multiple myeloma. J Clin Oncol (2011) 29(9):1125-32. doi:10.1200/JCO.2010.31.3304

52. Henry D, von Moos R, Vadhan-Raj S, Hungria V, Spencer A, Hirsh V, et al. A double-blind, randomized study of denosumab versus zoledronic acid for the treatment of bone metastases in patients with advanced cancer (excluding breast and prostate cancer) or multiple myeloma [Abstract 20LBA and Oral Presentation]. Eur J Cancer Suppl (2009) 7(3):11

53. Tabrizi MA, Tseng CM, Roskos LK. Elimination mechanisms of therapeutic monoclonal antibodies. Drug Discov Today (2006) 11:81-8. doi:10.1016/S13596446(05)03638-X

54. Wang W, Wang EQ, Balthasar JP. Monoclonal antibody pharmacokinetics and pharmacodynamics. Clin Pharmacol Ther (2008) 84:548-58. doi:10.1038/clpt. 2008.170

55. Scagliotti GV, Hirsh V, Siena S, Henry DH, Woll PJ, Manegold C, et al. Overall survival improvement in patients with lung cancer and bone metastases treated with denosumab versus zoledronic acid: subgroup analysis from a randomized, phase 3 study. J Thorac Oncol (2012) 7(2):1823-9. doi:10.1097/JTO.0b013e31826aec2b

56. Kitazawa S, Kitazawa R. RANK ligand is a prerequisite for cancer-associated osteolytic lesions. J Pathol (2002) 198:228-36. doi:10.1002/path.1199

57. Mundy GR. Metastasis to bone: causes, consequences, and therapeutic opportunities. Nat Rev Cancer (2002) 2:584-93. doi:10.1038/nrc867

58. Feeley BT, Liu NQ, Conduah AH, Krenek L, Roth K, Dougall WC, et al. Mixed metastatic lung cancer lesions in bone are inhibited by noggin overexpression and Rank: Fc administration. J Bone Miner Res (2006) 21:1571-80. doi:10.1359/jbmr.060706

59. Miller R, Jones J, Roudier M, et al. The RANKL inhibitor OPG-Fc either alone, or in combination with docetaxel, blocks lung cancer-induced osteolytic lesions or reduces skeletal tumor burden in a murine model of non-small cell lung cancer in bone. Presented at the 9th International Conference on Cancer-Induced Bone Disease, Arlington, VA (2009).

60. Miller RE, Roudier M, Jones J, Armstrong A, Canon J, Dougall WC. RANK ligand inhibition plus docetaxel improves survival and reduces tumor burden in a murine model of prostate cancer bone metastasis. Mol Cancer Ther (2008) 7:2160-9. doi:10.1158/1535-7163.MCT-08-0046

61. Gonzalez-Suarez E, Jacob AP, Jones J, Miller R, Roudier-Meyer MP, Erwert R, et al. RANK ligand mediates progestin-induced mammary epithelial proliferation and carcinogenesis. Nature (2010) 468:103-7. doi:10.1038/nature09495

62. Chen LM, Kuo CH, Lai TY, Lin YM, Su CC, Hsu HH, et al. RANKL increases migration of human lung cancer cells through intercellular adhesion molecule-1 up-regulation. J Cell Biochem (2011) 112:933-41. doi:10.1002/jcb.23009

63. Jones DH, Nakashima T, Sanchez OH, Kozieradzki I, Komarova SV, Sarosi I, et al. Regulation of cancer cell migration and bone metastasis by RANKL. Nature (2006) 440:692-6. doi:10.1038/nature04524

64. Peters S, Meylan E. Targeting receptor activator of nuclear factor-kappa B as a new therapy for bone metastases in non-small cell lung cancer. Curr Opin Oncol (2013) 25(2):137-44. doi:10.1097/CCO.0b013e32835d720b

65. Hirsh V. Bisphosphonates in lung cancer: can they provide benefits beyond prevention of skeletal morbidity? Anticancer Agents Med Chem (2012) 12(2):137-43. doi:10.2174/187152012799014922

66. Luo FR, Camuso A, McGlinchey K, Fager K, Flefleh C, Wen M-L, et al. Evaluation of anti-osteoclastic activity of the novel, oral multi-targeted kinase inhibitor Dasatinib (BMS-354825). Proceedings of the American Association for Cancer Research (2005), Molecular Targets and Cancer Therapeutics - Discovery, Biology, and Clinical Applications, AACR-NCI-EORTC International Conference; Nov 14-18; Philadelphia, PA. [Abstract B178] (2005). p. 173. [Poster Session B Antiangiogenic/Antivascular Agents].

67. Fields SZ, Parshad S, Anne M, Raftopoulos H, Alexander MJ, Sherman ML, et al. Activin receptor antagonists for cancer- related anemia and bone disease. Expert Opin Investig Drugs (2013) 22(1):87-101. doi:10.1517/13543784.2013. 738666

68. Borgstein NG, Yang Y, Condon CH, Haltom E, Sherman ML. ACE-011, a soluble activin receptor type IIA IgG-Fc fusion protein decreases follicle stimulating hormone and increases bone- specific alkaline phosphatase, a marker of bone formation in postmenopausal healthy women [abstract]. Cancer Res (2009) 69(2 Suppl 1):1160. doi:10.1158/0008-5472

69. Hellerstedt BA, Edelman G, Vogelzang NJ, Kluger HM, Yasenchak CA, Shen X, et al. Activitiy of cabozantinib (XL 184) in metastatic NSCLC: results from a phase II randomized discontinuation trial (RDT) [abstract]. J Clin Oncol (2012) 30(Suppl 15):7514.

70. Parker C, Nilsson S, Heinrich D, Helle SI, O’Sullivan JM, Fosså SD, et al. Alpha emitter radium-223 and survival in metastatic prostate cancer. $N$ Engl J Med (2013) 369(3):213-23. doi:10.1056/NEJMoa1213755 
Conflict of Interest Statement: The author declares that the research was conducted in the absence of any commercial or financial relationships that could be construed as a potential conflict of interest.

Received: 28 April 2014; paper pending published: 18 May 2014; accepted: 28 May 2014; published online: 16 June 2014.

Citation: Hirsh V (2014) Targeted treatments of bone metastases in patients with lung cancer. Front. Oncol. 4:146. doi: 10.3389/fonc.2014.00146
This article was submitted to Thoracic Oncology, a section of the journal Frontiers in Oncology.

Copyright (c) 2014 Hirsh. This is an open-access article distributed under the terms of the Creative Commons Attribution License (CC BY). The use, distribution or reproduction in other forums is permitted, provided the original author(s) or licensor are credited and that the original publication in this journal is cited, in accordance with accepted academic practice. No use, distribution or reproduction is permitted which does not comply with these terms. 